\title{
Adaptación basada en Ecosistemas en pequeñas fincas de granos básicos en Guatemala y Honduras ${ }^{1}$
}

\section{Ecosystem-based Adaptation by smallholder basic grain farms in Guatemala and Honduras}

\author{
Adina Chain-Guadarrama2 , M.R. Martínez-Rodríguez, J.M. Cárdenas ${ }^{4,5}$, S. Vílchez-Mendoza ${ }^{5}$, C.A. Harvey
}

\section{Resumen}

En Centroamérica, el maíz y el frijol son usualmente cultivados por agricultores que tienen pequeñas áreas de tierra y viven en situación de vulnerabilidad. El cambio climático representa una importante amenaza para estos pequeños agricultores de granos básicos, poniendo en peligro sus sistemas de producción y medios de vida. El uso de Adaptación basada en Ecosistemas (AbE) puede ayudarlos a enfrentar el cambio climático, pero existe poca información sobre el tema. El objetivo de este trabajo fue caracterizar las estrategias de AbE que los pequeños agricultores de granos básicos de cuatro paisajes de Guatemala y Honduras utilizan para aumentar su resiliencia frente al cambio climático. A través de entrevistas y mediciones en campo en 160 fincas, entre julio 2014 y junio $2015,1)$ se exploró qué tan común es el uso de las diferentes prácticas de AbE, 2) se documentaron las características biofísicas de estas prácticas, y 3) se determinó si la implementación y características de las prácticas difieren entre paisajes. Los resultados indican que muchos de los pequeños agricultores de granos básicos usan prácticas de AbE, aun cuando cultivan áreas pequeñas (parcelas de maíz de $\sim 0,68$ ha), tienen un bajo nivel de educación y bajo acceso a capacitaciones o consejos técnicos. Las prácticas más comunes fueron el uso de árboles dispersos, huertos caseros y cercas vivas. Resultados de un análisis de ANDEVA indican que hubo diferencias significativas en el uso de diferentes prácticas entre agricultores, que sugiere que las adoptan en respuesta a sus medios de vida y condiciones biofísicas. El estudio sugiere que los pequeños agricultores de granos básicos tienen la experiencia y el conocimiento para utilizar prácticas de AbE, y que ven beneficios derivados de su implementación; sin embargo, para ampliar el uso de estas prácticas, se necesita más apoyo técnico, financiero y político.

Palabras clave: cambio climático, cercos vivos, frijol, huertos caseros, maíz.

\begin{abstract}
In Central America maize and beans are usually cultivated by farmers who have small landholdings and live in vulnerable situations. Climate change is an important threat to these smallholder basic grain farmers, putting at risk

\footnotetext{
Recibido: 13 de marzo, 2018. Aceptado: 13 de junio, 2018. Este trabajo formó parte del proyecto CASCADA (Adaptación basada en Ecosistemas para pequeños agricultores de subsistencia y café en América Central), que forma parte de la Iniciativa Internacional por el Clima (ICI), financiada por el Ministerio Federal de Medio Ambiente, Protección de la Naturaleza, Obras públicas y Seguridad Nuclear (BMUB) del Gobierno de la República de Alemania.

2 Consultora independiente. Turrialba 30501, Costa Rica. achain@catie.ac.cr

3 Conservation International. 2011 Crystal Drive, Suite 500, Arlington, VA, 22202, USA. ruth.martinez@un.org, celiaharvey@stanfordalumni. org

4 Ministerio de Agricultura y Ganadería (MAG). Agencia de extensión de Turrubares, Costa Rica. jcardenas@mag.go.cr

5 Centro Agronómico Tropical de Investigación y Enseñanza (CATIE). Turrialba 30501, Costa Rica. svilchez@catie.ac.cr
} 
their production systems and their livelihoods. The use of Ecosystem-based Adaptation (EbA) can help them to adapt to climate change, however there is limited information on the use of EbA. The objective of the present work was to characterize the EbA strategies that smallholder basic grain farmers of four landscapes in Guatemala and Honduras use to increase their resilience to climate change. Using interviews and field measurements in 160 farms, between July 2014 and June 2015, we 1) explored how common the use of different EbA practices is, 2) documented the biophysical characteristics of these practices, and 3) explored if the implementation and characteristics of EbA practices differed among landscapes. Our results indicate that even though they own small land areas (mean maize plot area of $\sim 0.68$ ha), have low education levels and low access to technical training and advice, many smallholder basic grain farmers are using EbA practices in their farms. The most common EbA practices were the use of dispersed trees, home gardens and live fences. An ANOVA anlysis indicated significant differences in the use of different EbA practices among farmers, which suggests that farmers adapt practices in response to the social and biophysical conditions where they live. Our study suggests that smallholder basic grain farmers have the experience and the knowledge to use EbA practices and recognize the benefits derived from the implementation of such practices. However, in order to increase use of EbA practices, more technical, financial and political support is needed.

Keywords: beans, climate change, home gardens, live fences, maize.

\section{Introducción}

La producción de granos básicos es clave en la cultura e identidad de la agricultura centroamericana (García-Serrano y Del-Monte, 2004; Janick, 2013). Se estima que hay más de un millón de familias de pequeños agricultores que cultivan maíz (Zea mays) y frijol (Phaseolus vulgaris) para subsistencia a lo largo de la región, y que producen el 70\% del maíz y el 100\% del frijol que se consume localmente (Schmidt et al., 2012). La mayoría de los agricultores de granos básicos cultivan pequeñas áreas de tierra (típicamente con tamaños menores a dos hectáreas), obtienen bajos rendimientos de cultivos y viven en condiciones precarias (Eakin, 2000).

El cambio climático representa una importante amenaza para los medios de vida de los pequeños agricultores de granos básicos. En Centroamérica, el cambio climático está resultando en temperaturas más elevadas, cambios en los patrones de lluvia y cambios en la frecuencia e intensidad de eventos climáticos extremos (Imbach et al., 2017), todo lo cual tendrá impactos negativos sobre la producción de cultivos. Además, muchas de las áreas de producción de pequeños agricultores están expuestas a eventos de clima extremos, particularmente huracanes, inundaciones, lluvias torrenciales y sequías (Dilley et al., 2005).

Los pequeños agricultores de granos básicos dependen de la agricultura de secano, y son, por lo tanto, vulnerables a sequías prolongadas, patrones irregulares y eventos extremos de lluvia, los cuales afectan la siembra de cultivos, la cosecha y el rendimiento (Conde et al., 1997). La producción de maíz y frijol también es sensible a las altas temperaturas (particularmente las temperaturas nocturnas arriba de los $18{ }^{\circ} \mathrm{C}$ ), las cuales reducen la floración y la producción (Schmidt et al., 2012). Como resultado, es probable que muchos pequeños agricultores sufran reducciones significativas en los rendimientos de maíz y frijol, a menos que se pongan en práctica medidas de adaptación.

Un enfoque que podría ayudar a los pequeños agricultores de granos básicos para adaptarse al cambio climático es el uso de Adaptación basada en Ecosistemas (AbE) (Vignola et al., 2015). La AbE se refiere al uso de prácticas agrícolas que están basadas en la conservación, restauración y manejo sostenible de servicios ecosistémicos (SCBD, 2009). En parcelas de producción de maíz y frijol, las prácticas de AbE pueden incluir la plantación de cultivos en curvas de nivel, el uso de cultivos de cobertura o el establecimiento de terrazas para prevenir la erosión y pérdida de suelo durante eventos de lluvias torrenciales (Arriaza-Vallejo, 1995; Erenstein, 2003; Sivanpillai y Thurow, 
2008), y la inclusión de árboles en milpas para incrementar la retención de agua en condiciones de sequía y reducir los impactos de las altas temperaturas y los deslizamientos de tierra (Rocha, 1977; Hellin et al., 1999; FAO, 2005; Pérez-Marin et al., 2006). Otras prácticas de AbE incluyen el establecimiento de huertos caseros, para diversificar la producción de alimentos y minimizar pérdidas potenciales de cultivos ante eventualidades climáticas, el uso de cercas vivas para ayudar a reducir la erosión del suelo, la protección de bosques para mantener el suministro de agua y el uso de barbechos para asegurar la restauración y prevenir la erosión de los suelos por efecto de las lluvias torrenciales o huracanes (Vignola et al., 2015; Harvey et al., 2017).

Aun cuando hay un interés creciente para promover el uso de AbE para apoyar a los pequeños agricultores a adaptarse al cambio climático, hay poca información sobre cómo los pequeños agricultores de granos básicos están usando estas prácticas. El objetivo de este trabajo fue caracterizar las estrategias de AbE que los pequeños agricultores de granos básicos en cuatro paisajes de Guatemala y Honduras utilizan para aumentar su resiliencia frente al cambio climático.

\section{Materiales y métodos}

En este trabajo se caracterizaron las prácticas de $\mathrm{AbE}$ de pequeños agricultores de maíz y frijol, en un total de 160 fincas de cuatro paisajes centroamericanos: Acatenango ( $n=15$ fincas) y Chiquimula $(n=47)$ en Guatemala, y Choluteca $(\mathrm{n}=50)$ y Yoro $(\mathrm{n}=48)$ en Honduras (Figura 1). El área total abarcada por cada zona de estudio varió

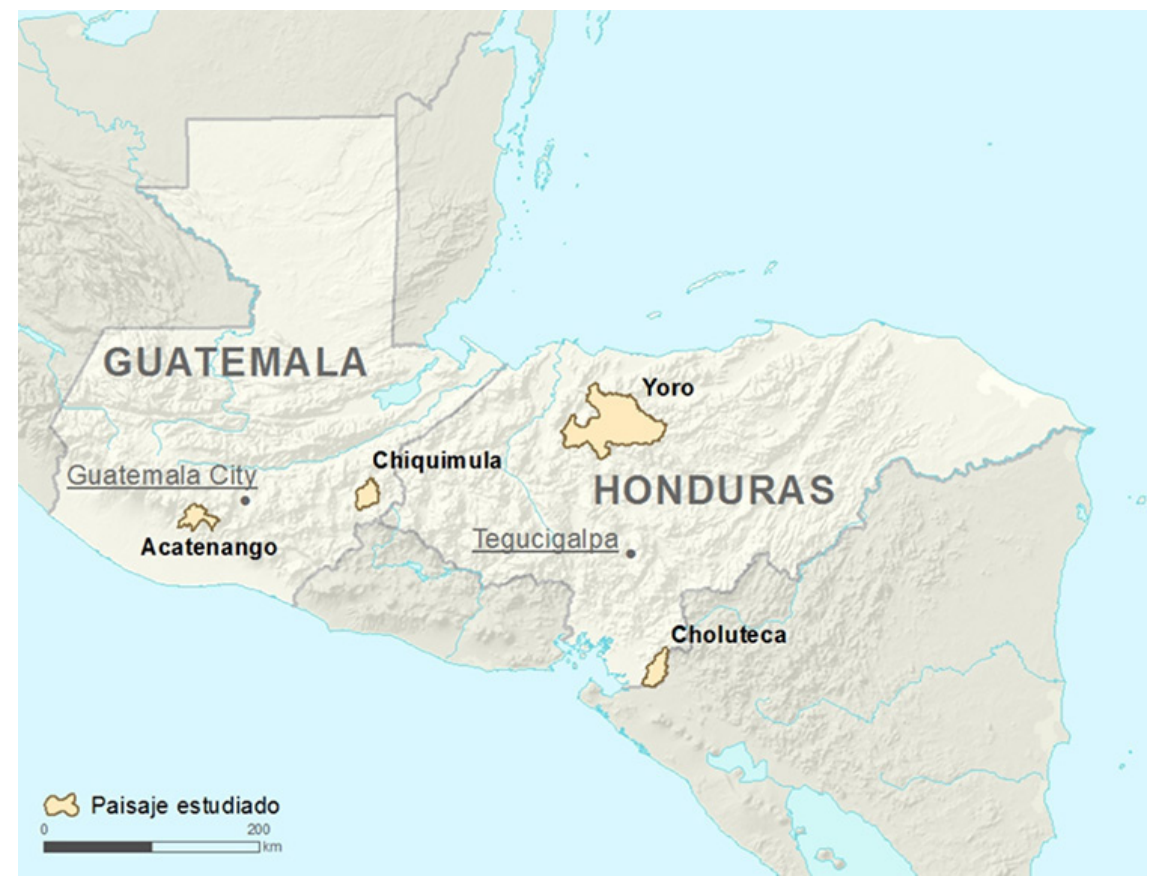

Figura 1. Ubicación de los paisajes agrícolas, dos en Guatemala y dos en Honduras, Centroamérica, donde se caracterizó el uso de prácticas de Adaptación basada en Ecosistemas (AbE) por pequeños agricultores de granos básicos. Julio 2014 - junio 2015.

Figure 1. Location of the agricultural landscapes, two in Guatemala and two in Honduras, Central America, in which the use of Ecosystem-based Adaptation (EbA) practices by smallholder basic grain farmers was characterized. July 2014 - June 2015. 
entre 39600 ha en Chiquimula y 325900 ha que abarca el paisaje de Yoro. Los paisajes están localizados según la clasificación Holdridge en zonas de vida contrastantes; bosque seco tropical en Choluteca, bosque húmedo subtropical en Chiquimula y Yoro, y bosque muy húmedo subtropical en Acatenango y Yoro. El bosque húmedo subtropical, generalmente distribuido en elevaciones entre 1000-2000 msnm, se caracteriza por una temperatura promedio anual que varía entre los $18-24{ }^{\circ} \mathrm{C}$ y una precipitación media anual entre los 1000-2000 mm. El bosque muy húmedo subtropical se distribuye en el mismo rango de elevación y temperatura, sin embargo, la precipitación media anual es más elevada, varía en un rango entre los 2000-4000 mm, está bien distribuida durante todo el año y se caracteriza por la ausencia de una estación seca bien marcada (Holdridge, 2000). El bosque seco tropical está asociado a una temperatura promedio anual superior a los $24^{\circ} \mathrm{C}$ y una precipitación media anual entre los 700-2000 $\mathrm{mmm}$, con uno o dos periodos marcados de sequía, que determina una periodicidad muy marcada en la fenología de la vegetación (Holdridge, 2000).

Las zonas de estudio están dominadas por fincas de pequeños agricultores que cultivan granos básicos, y representan comunidades con baja capacidad adaptativa al cambio climático (Holland et al., 2017). Las familias agricultoras se caracterizan por contar con un alto número de miembros por hogar $(\sim 5,5)$, con un bajo nivel educativo y bajo acceso a capacitación técnica (Cuadro 1$)$. Tienen fincas pequeñas $(\sim 1,75 \mathrm{ha})$, con un área

Cuadro 1. Características de los pequeños agricultores de granos básicos y sus fincas en cuatro paisajes de Guatemala y Honduras, en donde se describió el uso de prácticas de Adaptación basada en Ecosistemas (AbE). Julio 2014 - junio 2015.

Table 1. Characteristics of smallholder basic grain farmers and their farms in four landscapes in Guatemala and Honduras where Ecosystem-based Adaptation (EbA) practices were described. July 2014 - June 2015.

\begin{tabular}{|c|c|c|c|c|c|c|}
\hline & \multirow[t]{2}{*}{ Características } & $\begin{array}{l}\text { Acatenango, } \\
\text { Guatemala }\end{array}$ & $\begin{array}{l}\text { Chiquimula, } \\
\text { Guatemala }\end{array}$ & $\begin{array}{l}\text { Choluteca, } \\
\text { Honduras }\end{array}$ & $\begin{array}{c}\text { Yoro, } \\
\text { Honduras }\end{array}$ & Total \\
\hline & & $\mathrm{N}=15$ & $\mathrm{~N}=47$ & $\mathbf{N}=\mathbf{5 0}$ & $\mathrm{N}=48$ & $\mathrm{~N}=160$ \\
\hline \multirow{4}{*}{ 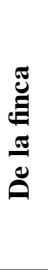 } & Tamaño (ha) promedio de todas las fincas & $2,11 \pm 0,10 \mathrm{a}$ & $2,05 \pm 0,12 \mathrm{ab}$ & $1,41 \pm 0,08 \mathrm{bc}$ & $1,00 \pm 0,10 \mathrm{c}$ & $1,75 \pm 0,01$ \\
\hline & $\begin{array}{l}\text { Tamaño promedio (ha) de las parcelas de } \\
\text { frijol }\end{array}$ & - & - & $0,13 \pm 0,02 b$ & $0,73 \pm 0,16 \mathrm{a}$ & $0,47 \pm 0,12$ \\
\hline & $\begin{array}{l}\text { Tamaño promedio (ha) de las parcelas de } \\
\text { maíz }\end{array}$ & $0,42 \pm 0,10 \mathrm{~b}$ & - & $0,58 \pm 0,05 b$ & $0,99 \pm 0,06 \mathrm{a}$ & $0,68 \pm 0,01$ \\
\hline & $\begin{array}{l}\text { Distancia promedio en } \mathrm{km} \text { de la casa la finca } \\
( \pm \mathrm{EE})\end{array}$ & $4,89 \pm 0,19 \mathrm{a}$ & $2,00 \pm 0,10 \mathrm{~b}$ & $1,04 \pm 0,10 \mathrm{c}$ & $1,32 \pm 0,10 \mathrm{bc}$ & $1,16 \pm 0,02$ \\
\hline \multirow{7}{*}{ 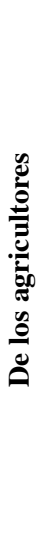 } & Tamaño promedio de la familia ( \pm EE) & $6,13 \pm 0,62 \mathrm{a}$ & $5,02 \pm 0,35$ a & $5,36 \pm 0,34 \mathrm{a}$ & $5,92 \pm 0,35$ a & $5,50 \pm 0,19$ \\
\hline & $\begin{array}{l}\text { Número promedio de años de experiencia } \\
\text { como agricultor }\end{array}$ & $27,53 \pm 4,25 \mathrm{a}$ & $31,28 \pm 2,40$ a & $32,76 \pm 2,33 \mathrm{a}$ & $31,08 \pm 2,37 \mathrm{a}$ & $31,33 \pm 1,29$ \\
\hline & $\begin{array}{l}\text { \# y }(\%) \text { de agricultores que recibieron } \\
\text { capacitación en los últimos dos años }\end{array}$ & $1(6,7)$ & $8(17,0)$ & $8(16,0)$ & $13(27,1)$ & $30(18,8)$ \\
\hline & $\begin{array}{l}\text { \# y }(\%) \text { de agricultores que recibieron } \\
\text { consejo de un agrónomo en los últimos dos } \\
\text { años }\end{array}$ & 0 & $1(2,1)$ & $9(18)$ & $6(12,5)$ & $16(10)$ \\
\hline & $\begin{array}{l}\text { \# y }(\%) \text { de agricultores que tienen menos que } \\
\text { el nivel de educación primaria completo }\end{array}$ & $13(86,7)$ & $34(72,3)$ & $33(66,0)$ & $41(85,4)$ & $121(75,6)$ \\
\hline & $\begin{array}{l}\text { \# y }(\%) \text { de agricultores que tienen solo el } \\
\text { nivel de educación primaria completo }\end{array}$ & $1(6,7)$ & $9(19,1)$ & $13(26,0)$ & $6(12,5)$ & $29(18,1)$ \\
\hline & $\begin{array}{l}\text { \# y }(\%) \text { de agricultores que tienen más que el } \\
\text { nivel de educación primaria completo }\end{array}$ & $1(6,7)$ & $4(8,5)$ & $4(8,0)$ & $1(2,1)$ & $10(6,3)$ \\
\hline
\end{tabular}

$\mathrm{N}=$ número de agricultores de granos básicos / N= number of basic grain farmers.

Se reportan medias ajustadas y errores estándar. Letras asociadas a las medias indican diferencias estadísticas entre paisajes, basado en ANDEVA $(\mathrm{p}<0,05) /$ Adjusted means and standard errors are reported. Letters associated with means indicate statistical differences across landscapes, based on ANOVA $(\mathrm{p}<0.05)$. 
promedio de parcela de cultivo de maíz de 0,68 ha y parcelas de cultivo de frijol con un tamaño promedio de 0,47 ha (Cuadro 1).

La selección de agricultores en cada paisaje se llevó a cabo con información previa obtenida a través de una encuesta realizada a 860 hogares de pequeños agricultores (Harvey et al., 2017). Específicamente, los agricultores fueron elegidos con base en información recolectada sobre el número de prácticas de AbE utilizada por finca, estratificándolos en dos grupos de alta y baja frecuencia de uso, y eligiendo al azar veinticinco agricultores de cada grupo, de manera que en cada paisaje hubiera una representación adecuada de la diversidad de fincas (Harvey et al., 2017).

Para caracterizar el uso de $\mathrm{AbE}$ en cada una de las fincas seleccionadas, primero se emplearon métodos de mapeo participativo con los agricultores, lo que permitió localizar parcelas individuales en la finca y caracterizar su uso de suelo (ej. campos de cultivo, potreros, barbechos, áreas forestales, cuerpos de agua, etc.). Posteriormente, se solicitó a los agricultores que identificaran cuáles prácticas de AbE tenían por parcela individual y que indicaran en el mapa su sitio de implementación, para poder visitar dichas áreas y caracterizar las prácticas a través de trabajo de campo.

Se registró la presencia/ausencia de once prácticas de AbE: el uso de árboles dispersos en parcelas de granos básicos, cercas vivas, huertos caseros, terrazas, surcos en contorno, cobertura viva, barbechos, bosques ribereños, parches de bosque y plantaciones forestales (Figura 2). Esta lista de prácticas de AbE estuvo basada en entrevistas con productores y expertos sobre prácticas comúnmente usada por pequeños agricultores de café y granos básicos (Harvey et al., 2017).
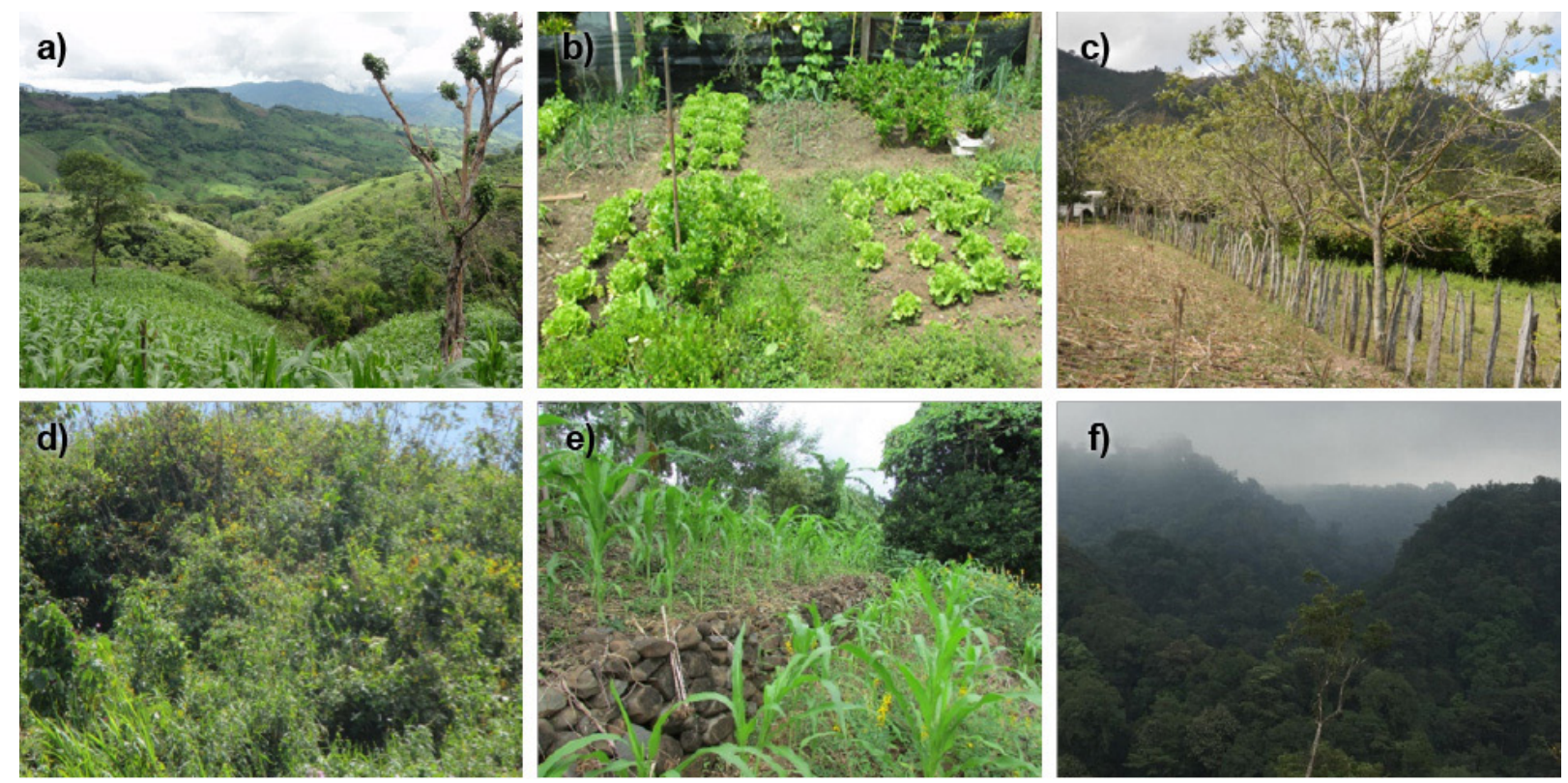

Figura 2. Prácticas de AbE utilizadas por pequeños agricultores de granos básicos en cuatro paisajes centroamericanos. a) árboles dispersos; b) huertos caseros; c) cercas vivas; d) barbecho; e) terrazas; y f) bosque. Guatemala - Honduras. Julio 2014 junio 2015.

Figure 2. EbA practices used by smallholder basic grain farmers in four Central American landscapes. a) dispersed trees; b) home gardens; c) live fences; d) fallows; e) terraces, and f) forest. Guatemala - Honduras. July 2014 - June 2015.

Durante julio de 2014 hasta junio de 2015, se colectó información detallada sobre el uso de cada práctica de AbE presente en la finca. Para bosques, bosques ribereños, huertos caseros y barbechos, se midió el área de todas 
las parcelas y se calculó el área total bajo esta práctica a nivel de la finca. La caracterización del uso de árboles dispersos dentro de las parcelas de granos básicos se realizó mediante la selección al azar de hasta tres parcelas por finca. Dentro de cada parcela se estableció un área de 20 x $50 \mathrm{~m}\left(100 \mathrm{~m}^{2}\right)$ en el centro, y se registraron todos los árboles dispersos, cuyo diámetro de altura de pecho (dap) fuera $>5 \mathrm{~cm}$, se identificó su especie y se midió su dap. Se utiliza el término común "árboles dispersos" para referirse al conjunto de especies de plantas registradas en estas parcelas que pueden incluir palmas u otras especies no leñosas. Por otra parte, se seleccionaron aleatoriamente hasta seis cercas vivas por finca y se registraron todos los individuos $>5 \mathrm{~cm}$ de dap dentro de un segmento de $100 \mathrm{~m}$ de la cerca elegido al azar, identificando sus especies y midiendo su dap.

En las dos prácticas analizadas la información se resumió en términos del promedio de especies por parcela, el dap promedio por parcela, y la densidad promedio (individuos/ha). También se registró si el agricultor usó o no cobertura viva, terrazas y surcos en contorno en las parcelas.

Todos los datos fueron colectados en campo, con el uso de tabletas programadas con SurveyCTO, un software que captura, registra y procesa los datos colectados durante entrevistas estructuradas y trabajo de campo.

Se empleó estadística descriptiva para caracterizar la prevalencia de las prácticas de AbE a través de las fincas y paisajes, calculando el porcentaje de fincas con prácticas de AbE individuales y el número total de estas por finca. También se describió la composición de especies de la vegetación dispersa en las parcelas de granos básicos y de la vegetación en las cercas vivas por paisaje. Se compararon los promedios de densidad arbórea, número de especies y diámetro arbóreo en las parcelas de granos básicos y cercas vivas, con base en el análisis de la varianza (ANDEVA). El paisaje de Acatenango no fue tomado en cuenta en estos análisis comparativos al presentar menos de cinco agricultores que implementaron cada una de estas prácticas. No fue posible comparar el área total de bosque, bosque ribereño, huertos caseros y barbechos, debido a tamaños de muestra muy pequeños $(\mathrm{n}<10)$ por paisaje. Todos los análisis se realizaron usando el paquete estadístico InfoStat (Di-Rienzo et al., 2017).

\section{Resultados}

\section{Uso de las prácticas de AbE por los pequeños agricultores}

En total, se caracterizaron 160 fincas de granos básicos, a través de la medición de 160 parcelas de maíz y/o frijol (incluyendo la medición de 1686 árboles dispersos), 160 segmentos de cercas vivas (con mediciones de 3143 árboles), 54 fragmentos de bosque (187,5 ha), 14 bosques ribereños (3,34 ha), 59 barbechos (49,3 ha) y una plantación forestal $(0,05 \mathrm{ha})$.

Los pequeños agricultores de granos básicos usaron en promedio 3,02 $\pm 0,07$ prácticas de $\mathrm{AbE}$, con un rango de 0 a 6 prácticas por finca. El paisaje con mayor número promedio de prácticas de $\mathrm{AbE}$ usadas por los agricultores fue Yoro, mientras que en Acatenango se observó el menor número de prácticas (Cuadro 2; $\mathrm{F}_{3,156}=28,22$, $\mathrm{p}<0,0001$ ). La práctica más común entre todos los agricultores fue el uso de árboles dispersos (72,5\% del total de agricultores), seguida por los huertos caseros $(68,8 \%)$, las cercas vivas $(53,1 \%)$ y los surcos en contorno (35\%) (Cuadro 2). Por el contrario, las prácticas menos comunes fueron el uso de terrazas, cobertura viva y la presencia de plantaciones forestales, en orden descendente, todas usadas por menos del 7\% de los agricultores de granos básicos (Cuadro 2). Además, se observaron diferencias en la frecuencia de uso de las prácticas entre paisajes. Por ejemplo en Chiquimula y Choluteca el porcentaje de agricultores que utilizaron árboles dispersos fue más del doble que aquellos en Yoro y Acatenango, mientras que, en estos dos últimos paisajes casi el 100\% de los agricultores tuvieron huertos caseros, menos del 10\% los reportaron en Acatenango. En general, Acatenango tuvo una baja frecuencia en el uso de prácticas de AbE. 
Cuadro 2. Número (y porcentaje) de pequeños agricultores de granos básicos que reportaron el uso de diferentes prácticas agrícolas de Adaptación basada en Ecosistemas, en cuatro paisajes de Guatemala y Honduras, Centroamérica. Julio 2014 - junio 2015.

Table 2. Number (and percent) of smallholder basic grain farmers reporting the use of different Ecosystem-based Adaptation practices in four landscapes of Guatemala and Honduras, in Central America. July 2014 - June 2015.

\begin{tabular}{lccccc}
\hline Prácticas de AbE* & $\begin{array}{c}\text { Acatenango, } \\
\text { Guatemala }\end{array}$ & $\begin{array}{c}\text { Chiquimula, } \\
\text { Guatemala }\end{array}$ & $\begin{array}{c}\text { Choluteca, } \\
\text { Honduras }\end{array}$ & $\begin{array}{c}\text { Yoro, } \\
\text { Honduras }\end{array}$ & $\begin{array}{c}\text { Total (4 } \\
\text { paisajes) }\end{array}$ \\
\hline \# y $(\%)$ de fincas & $15(\%)$ & $47(\%)$ & $50(\%)$ & $48(\%)$ & $160(\%)$ \\
Árboles dispersos en parcelas de granos básicos & $5(33,3)$ & $46(97,9)$ & $43(86)$ & $22(45,8)$ & $116(72,5)$ \\
Huertos caseros & $1(6,7)$ & $13(27,7)$ & $49(98)$ & $47(97,9)$ & $110(68,8)$ \\
Cercas vivas & $1(6,7)$ & $24(51,1)$ & $30(60)$ & $30(62,5)$ & $85(53,1)$ \\
Surcos en contorno & $10(66,7)$ & $11(23,4)$ & $8(16)$ & $27(56,3)$ & $56(35)$ \\
Bosque & & $7(14,9)$ & $11(22)$ & $25(52,1)$ & $43(26,9)$ \\
Barbecho & & $2(4,3)$ & $10(20)$ & $30(62,5)$ & $42(26,3)$ \\
Bosque ribereño & & & $5(10)$ & $9(18,8)$ & $14(8,8)$ \\
Terrazas & & $7(14,9)$ & $3(6)$ & $1(2,1)$ & $11(6,9)$ \\
Cobertura viva & & $1(2,1)$ & $4(8)$ & $1(2,1)$ & $5(3,1)$ \\
Plantación forestal & & & $3,26 \pm 0,17 \mathrm{~b}$ & $4,00 \pm 0,17 \mathrm{a}$ & $3,02 \pm 0,07$ \\
Número promedio de prácticas $( \pm$ E.E.) & $1,13 \pm 0,31 \mathrm{~d}$ & $2,36 \pm 0,18 \mathrm{c}$ & $3,0,6)$ \\
\hline
\end{tabular}

E.E.: error estándar / EE: standard error.

* Las prácticas se presentan en orden decreciente de abundancia / Practices are presented in decreasing order of abundance.

\section{Características de las prácticas de AbE usadas por los pequeños agricultores}

Los datos recolectados a través del trabajo en campo, permitieron caracterizar los árboles dispersos en las parcelas de granos básicos y la vegetación de las cercas vivas para los paisajes de Chiquimula, Choluteca y Yoro. En las 140 parcelas de granos básicos en donde se registró el uso de árboles dispersos, se observó una densidad promedio de 87,36 $( \pm 1,0)$ individuos por hectárea (Cuadro 3). La mayor densidad se observó en las fincas de Chiquimula con 116 individuos/ha en promedio, por arriba de la densidad observada en Choluteca y Yoro $\left(\mathrm{F}_{2,108}=5,9 ; \mathrm{p}=0,0037\right)$. Las parcelas de granos básicos de Chiquimula también obtuvieron los mayores valores promedio de riqueza de especies $\left(\mathrm{F}_{2,108}=16,1 ; \mathrm{p}<0,0001\right)$ en comparación con Choluteca y Yoro, los cuales tuvieron valores de riqueza de especies similares entre ellos (Cuadro 3). En promedio, los árboles dispersos en las parcelas de granos básicos en estos tres paisajes presentaron un diámetro a la altura del pecho (dap) de $16,6 \mathrm{~cm}( \pm 1,01)$, sin diferencias significativas entre ellos $\left(\mathrm{F}_{2,108}=2,2 ; \mathrm{p}=0,1148\right)$.

Entre los 1686 árboles dispersos medidos en todas las parcelas de granos básicos, se reconocieron 103 especies. La especie más común a través de todos los paisajes fue Cordia alliodora (de nombre común laurel), la cual correspondió al 17,1\% del total de árboles medidos (Cuadro 3). Las cuatro especies más frecuentes después del laurel fueron Spondias purpurea (jocote), una especie del género Lysiloma (quebracho), las especies Enterolobium cyclocarpum (guanacaste) y Gliricida sepium (madero negro), con entre 83 y 126 árboles medidos en todas las parcelas. Las especies más abundantes entre los árboles dispersos en las parcelas de granos básicos variaron entre los paisajes estudiados (Cuadro 3).

La mayoría de los árboles dispersos en las parcelas de granos básicos fueron reportados por los agricultores como árboles de servicio $(50,4 \%)$, debido a que brindan algún servicio ecosistémico, incluyendo sombra, materia orgánica, leña, refugio para la fauna, fijación de carbono, y belleza escénica, entre otros (Figura 3). Además, el 29,5\% correspondieron a árboles maderables y el 20\% a árboles frutales. En las fincas de Yoro se reportó el porcentaje más alto de árboles de servicio (74,6\%), muy por encima del 19,6\% de árboles reportados en Choluteca. 
Cuadro 3. Características de los árboles dispersos en parcelas productivas de granos básicos y la vegetación en cercas vivas presentes en fincas pequeños agricultores de granos básicos en tres paisajes, uno en Guatemala y dos en Honduras. Julio 2014 - junio 2015.

Table 3. Characteristics of dispersed trees in crop fields and vegetation in live fences present in smallholder basic grain farms in three landscapes, one from Guatemala and two from Honduras. July 2014 - June 2015.

\begin{tabular}{|c|c|c|c|c|c|}
\hline & & $\begin{array}{c}\text { Chiquimula, } \\
\text { Guatemala }\end{array}$ & Choluteca, Honduras & Yoro, Honduras & Total (3 paisajes) \\
\hline & Número de parcelas & 57 & 55 & 25 & 137 \\
\hline \multirow{5}{*}{ 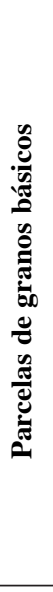 } & $\begin{array}{l}\text { Densidad arbórea } \\
\text { (árboles/ha) }\end{array}$ & $116,26 \pm 1,13 \mathrm{a}$ & $80,16 \pm 1,14 b$ & $56,52 \pm 1,20 \mathrm{~b}$ & $87,36 \pm 1,0$ \\
\hline & $\begin{array}{l}\text { Riqueza de especies } \\
\text { (por parcela) }\end{array}$ & $6,07 \pm 0,37 \mathrm{a}$ & $3,42 \pm 0,38 b$ & $3,18 \pm 0,53 b$ & $4,47 \pm 0,13$ \\
\hline & $\begin{array}{l}\text { Diámetro de árboles } \\
(\mathrm{dap} ; \mathrm{cm})\end{array}$ & $18,69 \pm 1,08 \mathrm{a}$ & $15,54 \pm 1,08 \mathrm{a}$ & $14,53 \pm 1,12 \mathrm{a}$ & $16,61 \pm 1,01$ \\
\hline & Número de individuos & 887 & 530 & 240 & 1686 \\
\hline & $\begin{array}{l}\text { Cinco especies más } \\
\text { comunes }(\%)\end{array}$ & $\begin{array}{c}\text { Spondias purpurea } \\
(14,0) ; \text { Swietenia } \\
\text { humilis }(9,1) ; \\
\text { Gliricidia sepium }(8,0) ; \\
\text { Schizolobium parahyba } \\
(7,6) ; \text { Lysiloma } \text { sp. } \\
(6,9) ; \text { Byrsonima } \\
\text { crassifolia }(5,3)\end{array}$ & $\begin{array}{c}\text { Cordia alliodora } \\
(47,6) ; \text { Enterolobium } \\
\text { cyclocarpum }(17,2) ; \\
\text { Lysiloma sp. }(7,0) ; \\
\text { Swietenia macrophylla } \\
(4,2) ; \text { Anacardium } \\
\text { occidentale }(3,8) ; \\
\text { Crescentia alata }(2,6) ; \\
\text { Tabebuia guayacan } \\
\end{array}$ & $\begin{array}{l}\text { Inga } \text { sp. }(16,3) ; \text { Mulule } \\
(16,3) ; \text { Cordia alliodora } \\
(7,9) ; \text { Lysiloma } \text { sp. } \\
(7,1) ; \text { Byrsonima } \\
\text { crassifolia }(7,1) ; \text { Persea } \\
\text { americana }(6,7)\end{array}$ & $\begin{array}{l}\text { Cordia alliodora }(17,1) ; \\
\text { Spondias purpurea } \\
(7,5) ; \text { Lysiloma } \mathrm{sp} . \\
(6,8) ; \text { Enterolobium } \\
\text { cyclocarpum }(5,5) ; \\
\text { Gliricidia sepium }(4,9) ; \\
\text { Swietenia humilis }(4,8)\end{array}$ \\
\hline & Número de segmentos & 41 & 57 & 59 & 157 \\
\hline \multirow{5}{*}{ 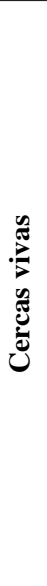 } & $\begin{array}{l}\text { Densidad arbórea } \\
\text { (árboles/ha) }\end{array}$ & $232,95 \pm 1,18 b$ & $170,89 \pm 1,16 b$ & $408,98 \pm 1,16 \mathrm{a}$ & $254,68 \pm 1,04$ \\
\hline & $\begin{array}{l}\text { Riqueza de especies } \\
\text { (por parcela) }\end{array}$ & $4,63 \pm 0,35 \mathrm{a}$ & $1,87 \pm 0,32 \mathrm{~b}$ & $2,20 \pm 0,32 b$ & $2,77 \pm 0,13$ \\
\hline & $\begin{array}{l}\text { Diámetro de árboles } \\
(\mathrm{dap} ; \mathrm{cm})\end{array}$ & $15,29 \pm 1,09 \mathrm{a}$ & $10,03 \pm 1,08 \mathrm{~b}$ & $8,15 \pm 1,08 b$ & $10,49 \pm 1,03$ \\
\hline & Número de individuos & 600 & 744 & 1765 & 3143 \\
\hline & $\begin{array}{l}\text { Cinco especies más } \\
\text { comunes }(\%)\end{array}$ & $\begin{array}{c}\text { Yucca guatemalensis } \\
(26,8) ; \text { Erythrina } \\
\text { sp. (Grupo de E, } \\
\text { costaricensis y E, } \\
\text { berteroana) }(20,2) ; \\
\text { Bursera simaruba } \\
(11,3) ; \text { Gliricidia } \\
\text { sepium }(6,3) ; \text { Spondias } \\
\text { purpurea }(5,2)\end{array}$ & $\begin{array}{c}\text { Cordia dentata }(92,7) ; \\
\text { Cordia alliodora }(1,6) ; \\
\text { Guazuma ulmifolia } \\
(1,1) ; \text { Gliricidia sepium } \\
(1,0) ; \text { Azadirachta } \\
\text { indica }(0,5)\end{array}$ & $\begin{array}{c}\text { Yucca guatemalensis } \\
(77,6) ; \text { Jatropha curcas } \\
(7,0) ; \text { Gliricidia sepium } \\
(5,8) ; \text { Bursera simaruba } \\
(5,1) ; \text { Perymenium } \\
\text { grande }(0,9)\end{array}$ & $\begin{array}{c}\text { Yucca guatemalensis } \\
(49,7) ; \text { Cordia dentata } \\
(22,0) ; \text { Bursera } \\
\text { simaruba }(5,1) ; \\
\text { Gliricidia sepium }(4,7) ; \\
\text { Jatropha curcas }(4,0)\end{array}$ \\
\hline
\end{tabular}

Letras asociadas a las medias indican diferencias estadísticas entre paisajes, basado en ANDEVA $(\mathrm{p}<0,05) /$ Letters associated with means indicate statistical differences across landscapes, based on ANOVA $(\mathrm{p}<0.05)$.

Finalmente, el porcentaje de árboles maderables en Choluteca fue más del doble que en Yoro y Chiquimula, y el porcentaje de árboles frutales en Chiquimula más del doble que en Choluteca y Yoro (Figura 3).

Con respecto a la caracterización de las cercas vivas, estas presentaron en promedio una densidad de 254,68 $( \pm 1,04)$ individuos $/ \mathrm{km}$ (Cuadro 3$)$. En este caso, las cercas vivas del paisaje de Yoro tuvieron una mayor densidad de individuos/ $\mathrm{km}$ en comparación con el resto de los paisajes $\left(\mathrm{F}_{2,81}=9,36 ; \mathrm{p}=0,0002\right)$ (Cuadro 3). Sin embargo, la alta densidad observada en las cercas vivas en las fincas de Yoro no estuvo reflejada en la riqueza de especies, ya que estas tuvieron el menor número en comparación con el resto de los paisajes $\left(\mathrm{F}_{2.81}=19,40 ; \mathrm{p}<0,0001\right)$, siendo las cercas vivas 

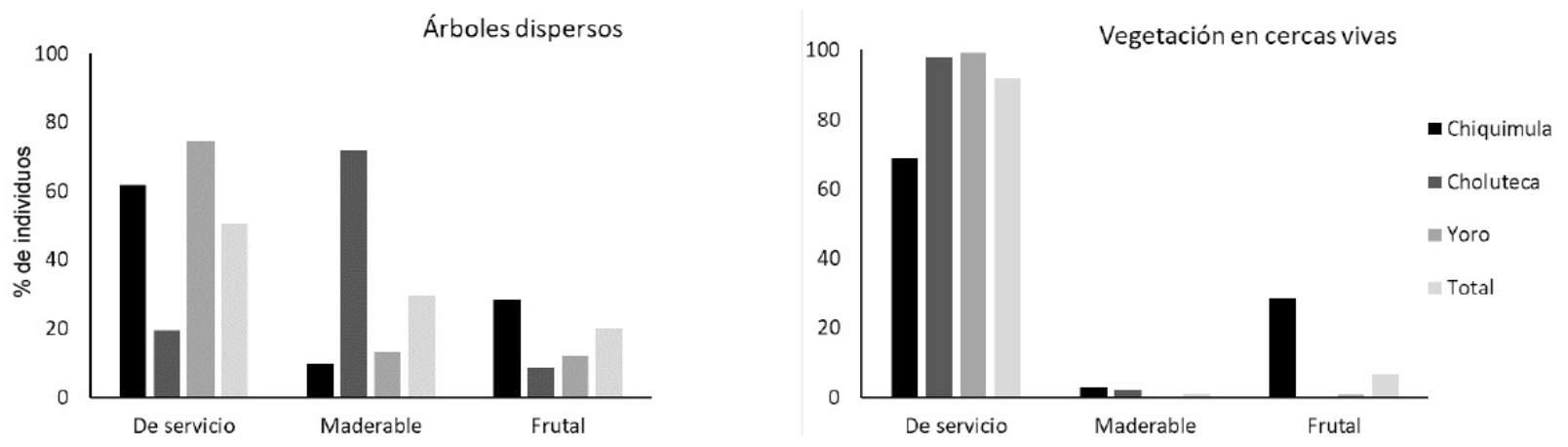

Figura 3. Uso de árboles dispersos registrados en las parcelas de granos básicos ( $n=1686)$ y las cercas vivas (n=3143) en fincas de pequeños agricultores de granos básicos en tres paisajes centroamericanos, uno en Guatemala (Chiquimula) y dos en Honduras (Choluteca y Yoro). Julio 2014 - junio 2015.

Figure 3. Use of dispersed trees present in basic grain fields $(n=1686)$ and live fences $(n=3143)$ in smallholder basic grain farms in three Central America landscapes, one in Guatemala (Chiquimula) and two in Honduras (Choluteca and Yoro). July 2014 - June 2015.

más diversas aquellas presentes en las fincas de Chiquimula (Cuadro 3). Los individuos de las cercas vivas estudiadas tuvieron diámetros promedios menores que los árboles dispersos registrados en las parcelas de café, con 10,49 cm $( \pm 1,03)$ de dap en todos los paisajes. En general, los individuos de las cercas vivas en Chiquimula tuvieron diámetros mayores que en Choluteca y Yoro $\left(\mathrm{F}_{2,81}=16,2 ; \mathrm{p}<0,0001\right)$.

Entre los 3143 árboles individuales medidos en las cercas vivas se reconocieron 77 especies. La especie más común fue Yucca guatemalensis (itabo o izote), con el 49,7\% de los individuos medidos. Las especies Cordia dentata (tigüilote) y Bursera simaruba (indio desnudo, jiote o mulato) fueron las segundas especies más frecuentes después del itabo, con 690 y 161 individuos medidos en todas las parcelas, respectivamente (Cuadro 3). Entre otras especies comunes presentes en las cercas vivas de los pequeños agricultores de granos básicos también se observaron el madero negro y la Jatropha curcas (piñón o jatrofa). Las especies más abundantes en las cercas vivas variaron entre los paisajes estudiados (Cuadro 3).

\section{Discusión}

Muchos de los pequeños agricultores de granos básicos de la región usan prácticas de Adaptación basada en Ecosistemas (AbE) en sus fincas, su uso y características variaron entre paisajes. Se ha demostrado que diferentes factores biofísicos y socioeconómicos como son el tamaño de la finca (Ithinji, 2011), la edad de los agricultores (Thangata y Alavalapti, 2003; Hisali et al., 2011), el nivel de educación (Knowler y Bradshaw, 2007; Deressa et al., 2011; Below et al., 2012) y la tenencia de la tierra (Erenstein, 2003; Hellin y Schrader, 2003; Hisali et al., 2011; Wall, 2007; Hellin y López-Ridaura, 2016), pueden estar asociados al uso y tipo de prácticas implementadas por pequeños agricultores en otras regiones.

Aunque más estudios son requeridos en la región centroamericana para entender qué factores estarían influenciando la selección de prácticas de AbE por pequeños agricultores de granos básicos, es probable que las diferencias en el uso de las mismas entre los agricultores en diferentes regiones reflejen tanto diferencias biofísicas (ej. el tipo de terreno, tamaño de la finca) como socioeconómicas (ej. el nivel de educación y acceso a capacitaciones). Por ejemplo, Acatenango y Yoro son paisajes más montañosos que el resto y fueron también en donde se reportó con mayor frecuencia el uso de surcos en contorno. Acatenango y Yoro fueron respectivamente 
los paisajes con el menor y el mayor porcentaje de agricultores que recibían algún tipo de consejo o capacitación técnica. En ese mismo sentido, Acatenango fue donde se reportó el menor número de prácticas y en Yoro donde los agricultores hicieron mayor uso de las mismas, lo que sugiere que las capacitaciones técnicas podrían ser importantes en la promoción de estas. El poco uso de otras prácticas de AbE, incluyendo la conservación de parches de bosque o áreas de barbecho, probablemente estuvo relacionado al hecho de que estas ocupan un área de terreno significativa y, por lo tanto, representan un alto costo de oportunidad a los finqueros con terrenos pequeños (Current, 1995; Michalski et al., 2010). La baja frecuencia en el uso de cobertura viva, ampliamente promovido pero poco implementado, podría estar explicada por la mano de obra adicional requerida (especialmente para su establecimiento), el costo de las semillas de la cobertura viva y la necesidad de cambiar prácticas de manejo de la finca (Méndez et al. 1998; Erenstein, 2003; Hellin y López-Ridaura, 2016).

Los resultados de esta investigación tienen varias implicaciones importantes esfuerzos locales y nacionales para impulsar el uso de AbE para apoyar a los pequeños agricultores a adaptarse al cambio climático. Primero, el número y variedad de prácticas de AbE implementadas en las fincas estudiadas, sugiere que los agricultores de granos básicos tienen la experiencia y el conocimiento para utilizarlas y que ven beneficios derivados de su implementación. En particular, la abundancia de algunas de ellas, como árboles dispersos, cercas vivas y huertos caseros, probablemente no solo refleja sus beneficios de adaptación sino que muchas de ellas, reconocidas ahora como $\mathrm{AbE}$, son prácticas agroecológicas que las poblaciones locales han usado por décadas para mejorar los suelos, y aumentar la productividad, y recibir otros cobeneficios como leña y subproductos (ej. Altieri et al., 2012). El intercambio de conocimiento de campesino a campesino dentro o entre regiones, podría ayudarles a comprender y adaptar el uso de prácticas poco utilizadas en sus regiones, y/o mejorar el uso de aquellas que ya implementan. Se ha demostrado que diferentes experiencias de aprendizaje participativo son efectivas para promover la adopción de nuevas prácticas en otros lados (Braun et al., 2000; Holt-Giménez, 2002).

Otra implicación de este trabajo acerca de los esfuerzos necesarios para promocionar la implementación de $\mathrm{AbE}$, es que demuestra que existe el potencial para incrementar y/o diversificar el uso de estas prácticas en las zonas estudiadas. Tal es el caso particular de Acatenango, en donde se reportó muy bajo uso de prácticas de AbE, a pesar de su gran necesidad de adaptación al ubicarse en el arco seco de Centroamérica (Imbach et al., 2017). De igual manera, existe la posibilidad de impulsar el uso de algunas prácticas de AbE que no son actualmente comunes, como la conservación de bosques ribereños, por medio de campañas de educación sobre la importancia de estos elementos para la conservación de agua y a través de incentivos para promover esta práctica en fincas de pequeños agricultores. La creación de capacitaciones por medio de actividades participativas, y los incentivos financieros son ejemplos que podrían mejorar la adopción de prácticas (Tucker et al., 2010; Deressa et al., 2011; Bryan et al., 2013).

Las diferencias observadas entre paisajes en las características de densidad, tamaño, composición y riqueza de árboles dispersos y cercas vivas en las parcelas de granos básicos, deberían ser tomadas en cuenta para asegurarse que las especies promovidas por proyectos de adaptación estén adecuadas a las condiciones biofísicas del paisaje y sean aceptadas por los agricultores en función de sus necesidades (i.e. por aquellos servicios adicionales que las prácticas puedan brindar) y mano de obra disponible para su mantenimiento. Esto es particularmente importante, ya que el uso de la práctica y sus características, en parte, responden a la promoción que de ellas hacen los servicios de extensión agrícola, cooperativas de agricultores y organizaciones no gubernamentales, por su habilidad para mejorar la sustentabilidad de la finca, apoyar la producción agrícola y mejorar los medios de vida de los finqueros (Harvey et al., 2017). Otros estudios han también señalado la importancia de seleccionar cuidadosamente las especies de árboles que son apropiadas para contextos biofísicos particulares y objetivos específicos, por ejemplo el uso de cercas vivas puede ser promovido como una manera efectiva de dividir campos, crear barreras para el movimiento animal y proveer forraje para animales, leña, madera y frutos (Harvey et al., 2005). 


\section{Conclusiones}

Muchos pequeños agricultores de granos básicos hacen uso de prácticas de AbE, particularmente árboles dispersos en las parcelas de maíz y/o frijol y huertos caseros, con un promedio de tres prácticas por finca. Sin embargo, el uso de AbE varía entre agricultores de diferentes paisajes y contextos, y existen diferencias en cómo estas prácticas son implementadas.

Este estudio es el primero en examinar el uso de prácticas de AbE por pequeños agricultores, y sirve como línea base, primero para evaluar la prevalencia y uso de prácticas particulares en diferentes regiones, y segundo para guiar a responsables de políticas y profesionales interesados en promover un mayor uso de AbE, particularmente en regiones en donde existe una baja o nula frecuencia de las mismas. El potencial de estas prácticas basadas en la conservación, restauración y manejo sostenible de servicios ecosistémicos para apoyar en estrategias de adaptación al cambio climático, está asociado por ejemplo al mejoramiento que otorgan en procesos de regulación hídrica y de erosión del suelo, a la reducción de los impactos de altas temperaturas y a la reducción de pérdidas de cultivos ante eventualidades climáticas. Es por ello que, el conocimiento acerca de las ventajas, desventajas, beneficios adaptativos y requerimientos de mano de obra, entre otros, que los agricultores y técnicos que hacen uso de estas prácticas tienen, debe ser documentado y diseminado, con el propósito de propiciar la adopción de las mismas y ayudar a los pequeños agricultores a tomar las medidas necesarias para adaptarse al cambio climático.

\section{Agradecimientos}

Los autores agradecen a Jacques Avelino, Bruno Rapidel, Raffaele Vignola y a Camila Donatti por sus contribuciones al diseño de la investigación, Kellee Koenig por ayuda en la preparación de la Figura 1, Nishina Nambiar por colaborar en la búsqueda de literatura y a Vlasova González por su apoyo logístico y administrativo.

\section{Literatura citada}

Altieri, M.A., F.R. Funes-Monzot, and P. Petersen. 2012. Agroecologically efficient agricultural systems for smallholder farmers: contributions to food sovereignty. Agron. Sustain. Dev. 32:1-13. doi:10.1007/s13593-011-0065-6

Arriaza-Vallejo, N.A. 1995. Comparación de tres prácticas agronómicas para el manejo y conservación de suelos en ladera, bajo el sistema frijol-maíz en Turrialba, Costa Rica. Tesis M.Sc., CATIE, Turrialba, CRC.

Below, T.B., K.D. Mutabazi, D. Kirschke, C. Franke, S. Sieber, R. Siebert, and K. Tscherning. 2012. Can farmers' adaptation to climate change be explained by socio-economic household-level variables? Glob. Environ. Change 22 :223-235. doi:10.1016/j.gloenvcha.2011.11.012

Braun,A.R., G. Thiele, and M. Fernández. 2000. Farmer field schools and local agricultural research committees: complementary platforms for integrated decision-making in sustainable agriculture. Network Paper $\mathrm{N}^{\circ} 105$. Agricultural Research and Extension Network, London, GBR. https://www.odi.org/sites/odi.org.uk/files/odi-assets/publications-opinion-files/8195. pdf (accessed 11 Jun. 2018).

Bryan, E., C. Ringler, B. Okoba, C. Roncoli, S. Silvestri, and M. Herrero. 2013. Adapting agriculture to climate change in Kenya: household strategies and determinants. J. Environ. Manage. 114:26-35. doi:10.1016/j.jenvman.2012.10.036

Conde, C., D. Liverman, M. Flores, R. Ferrer, R. Araújo, E. Betancourt, G. Villareal, and C. Gay. 1997. Vulnerability of rainfed maize crops in Mexico to climate change. Clim. Res. 9:17-23. doi:10.3354/cr009017 
Current, D., 1995. Economic and institutional analysis of projects promoting on farm tree planting in Costa Rica. En: D. Current et al., editors, Costs, benefits and farmers' adoption of agroforestry-project experience in Central America and the Caribbean. World Bank Publications, WA, USA. p. 45-71.

Deressa, T.T., R.M. Hassan, and C. Ringler. 2011. Perception of and adaptation to climate change by farmers in the Nile basin of Ethiopia. J. Agric. Sci. 149:23-31. doi:10.1017/S0021859610000687

Dilley, M., R.S. Chen, U. Deichmann, A.L. Lerner-Lam, M. Arnold, J. Agwe, P. Buys, O. Kjevstad, B. Lyon, and G. Yetman, 2005. Natural disaster hotspots: A global risk analysis. Rep. 34423. World Bank, WA, USA.

Di-Rienzo J.A., F. Casanoves, M.G. Balzarini, L. Gonzalez, M. Tablada, y C.W. Robledo. 2017. InfoStat versión 2017. Grupo InfoStat, Universidad Nacional de Córdoba, ARG.

Eakin, H., 2000. Smallholder maize production and climatic risk: A case study from Mexico. Clim. change 45:19-36. doi:10.1023/A:1005628631627

Erenstein, O. 2003. Smallholder conservation farming in the tropics and sub-tropics: a guide to the development and dissemination of mulching with crop residues and cover crops. Agric. Ecosyst. Environ. 100:17-37. doi:10.1016/S01678809(03)00150-6

FAO. 2005. El sistema agroforestal Quesungual. FAO, Roma, ITA.

García-Serrano, C.R., and J.P. Del-Monte. 2004. The use of tropical forest (agroecosystems and wild plant harvesting) as a source of food in the bribri and cabecar cultures in the Caribbean Coast of Costa Rica. Econ. Bot. 58:58-71. doi:10.1663/00130001(2004)058[0058:TUOTFA]2.0.CO;2

Harvey, C.A., M.R. Martínez-Rodríguez, J.M. Cárdenas, J. Avelino, B. Rapidel, R. Vignola, C.I. Donatti, and S. VilchezMendoza. 2017. The use of Ecosystem-based Adaptation practices by smallholder farmers in Central America. Agric. Ecosyst. Environ. 246:279-290. doi:10.1016/j.agee.2017.04.018.

Harvey, C.A., C. Villanueva, J. Villacís, M. Chacón, D. Muñoz, M. López, M. Ibrahim, R. Gómez, R. Taylor, J. Martínez, A. Navas, J. Saenz, D. Sánchez, A. Medina, S. Vilchez, B. Hernández, A. Perez, F. Ruiz, F. López, I. Lang, and F.L. Sinclair. 2005. Contribution of live fences to the ecological integrity of agricultural landscapes. Agric. Ecosyst. Environ. 111:200230. doi:10.1016/j.agee.2005.06.011

Hellin, J., and S. López-Ridaura. 2016. Soil and water conservation on Central American hillsides: if more technologies is the answer, what is the question? AIMS Agric. Food 1:194-207. doi:10.3934/agrfood.2016.2.194

Hellin, J., and K. Schrader. 2003. The case against direct incentives and the search for alternative approaches to better land management in Central America. Agric. Ecosyst. Environ. 99:61-81. doi:10.1016/S0167-8809(03)00149-X

Hellin, J., L.A., Welchez, and I. Cherrett. 1999. The quezungual system: an indigenous agroforestry system from western Honduras. Agrofor. Syst. 46:229-237. doi:10.1023/A:1006217201200

Hisali, E., P. Birungi, and F. Buyinza. 2011. Adaptation to climate change in Uganda: evidence from micro level data. Glob. Environ. Change 21:1245-1261. doi:10.1016/j.gloenvcha.2011.07.005

Holdridge, L.R. 2000. Ecología basada en zonas de vida. 2a ed. IICA. San José, CRC.

Holland, M.B., S.Z. Shamer, P. Imbach, J.C. Zamora, C. Medellin, E.J. Leguia, C.I. Donatti, M.R. Martínez-Rodríguez, and C.A. Harvey. 2017. Mapping agriculture and adaptive capacity: applying expert knowledge at the landscape scale. Clim. Change 141:139-153. doi:10.1007/s10584-016-1810-2

Holt-Giménez, E. 2002. Measuring farmers' agroecological resistance after Hurricane Mitch in Nicaragua: a case study in participatory, sustainable land management impact monitoring. Agric. Ecosyst. Environ. 93:87-105. doi:10.1016/S01678809(02)00006-3 
Imbach, P., M. Beardsley, C. Bouroncle, C. Medellin, P. Läderach, H. Hidalgo, E. Alfaro, J. VanEtten, R. Allan, D. Hemming, R. Stone, L. Hannah, and C.I. Donatti. 2017. Climate change, ecosystems and smallholder agriculture: An introduction to the special issue. Clim. Change 141:1-12. doi:10.1007/s10584-017-1920-5

Ithinji, G.K. 2011. Determinants of adaptation of shade coffee technology and the role of agroforestry in the productivity and profitability of coffee in South District, Kenya. PhD. Thesis, Egerton University, Nairobi, KEN.

Janick, J. 2013. Development of new world crops by indigenous Americans. Hort. Sci. 48:406-412.

Knowler, D., and B. Bradshaw. 2007. Farmers' adoption of conservation agriculture: a review and synthesis of recent research. Food Policy 21:25-48. doi:10.1016/j.foodpol.2006.01.003

Méndez, MA., O. Cáceres, P. Galeano, R.F. Hernández, y R.M. Zavala. 1998. Efecto del asocio de maíz con dos especies de frijol abono Mucuna pruriens Bort y Cannavalia ensiformis 1.d.c. en las poblaciones de plagas del suelo y follaje en Miraflor, Estelí, Nicaragua. En: O. Caceres, editor, Compartiendo una experiencia de manejo integrado de plagas: PROMIPPAC / Fase 95-98. EPAEZ; COSUDE, y PROMIPPAC, Managua, NIC. p. 112-116.

Michalski, F., J.P. Metzger, and C.A. Peres. 2010. Rural property size drives patterns of upland and riparian forest retention in a tropical deforestation frontier. Glob. Environ. Change 20:705-712. doi:10.1016/j.gloenvcha.2010.04.010

Pérez-Marin, A.M., R.S. César-Menezes, E. Dias-Silva, e E. Valadares-de-Sá-Barreto-Sampaio. 2006. Efeito da Gliricidia sepium sobre nutrientes do solo, microclima e produtividade do milho em sistema agroflorestal no agreste paraibano. Rev. Bras. Ciênc. Solo 30:555-564. doi:10.1590/S0100-06832006000300015

Rocha, J.A.N. 1977. Erosión de suelos de pendientes cultivadas con maíz y frijol con diferentes grados de cobertura viva dentro de una plantación forestal. Tesis M.Sc., CATIE, Turrialba, CRC.

SCBD (Secretariat of the Convention on Biological Diversity). 2009. Connecting biodiversity and climate change mitigation and adaptation: report of the second Ad Hoc technical expert group on biodiversity and climate change. Rep. 41. CBD, Montreal, CAN.

Sivanpillai, R., and T.L. Thurow. 2008. Hazard management lessons learned through mapping and assessing landslides triggered by Hurricane Mitch. In: Proceedings of the 15th International Congress of the International Soil Conservation Organization. Budapest, HUN. ISCO. http://tucson.ars.ag.gov/isco/isco15/pdf/Silvanpillai\%20R\%20Thurow\%20T_ Hazard\%20management\%20lessons.pdf (accessed Jun. 11, 2018).

Schmidt, A., A. Eitzinger, K. Sonder, y G. Sain. 2012. Tortillas on the roaster: Central America's maize-bean systems and the changing climate. Rep. 6. CIAT Policy, Cali, COL.

Thangata, P.H., and J.R.R. Alavalapati. 2003. Agroforestry adoption in southern Malawi: the case of mixed intercropping of Gliricidia sepium and maize. Agric. Syst. 78:57-71. doi:10.1016/S0308-521X(03)00032-5

Tucker, C.M., H. Eakin, and E.J. Castellanos. 2010. Perceptions of risk and adaptation: coffee producers market shocks, and extreme weather in Central America and Mexico. Glob. Environ. Change 20:23-32. doi:10.1016/j.gloenvcha.2009.07.006

Vignola, R., C.A. Harvey, P. Bautista-Solis, J. Avelino, B. Rapidel, C.I. Donatti, and M.R. Martínez. 2015. Ecosystem-based adaptation for smallholder farmers: definitions, opportunities and constraints. Agriculture. Agric. Ecosyst. Environ. 211:126-132. doi:10.1016/j.agee.2015.05.013.

Wall, P.C. 2007. Tailoring conservation agriculture to the needs of small farmers in developing countries: an analysis of issues. J. Crop Improv. 19:137-155. doi:10.1300/j411v19n01_07 